\title{
A control model for assembly manufacturing systems
}

\author{
A. B. Dolgui
}

Belarussian State University of Informatics and Radio Electronics

6, P. Brovki Street 220027 Minsk, Belarus

CRIN - Ecole des Mines de Nancy, 54042 Nancy Cedex, France

Phone 83.58.41.77 fax83.57.97.94 e-mail dolguia@loria.fr

M. C. Portmann

CRIN - Ecole des Mines de Nancy, 54042 Nancy Cedex, France

Phone 83.58.41.85 fax 83.57.97.94 e-mail portmann@loria.fr

J. M. Proth

INRIA - Lorraine, Project SAGEP

CESCOM, Technopole Metz, 2000, 57070 Metz, France

Phone 87.20.35.00 fax 87.76.39.77 e-mail proth@ilm.loria.fr

\begin{abstract}
In this paper, medium term planning problems related to multi-product assembly manufacturing systems are considered. The assembly lines are fed by external suppliers. Their delivery delays, not known in advance, are crucial for the assembly process. If some required components are not available at assembly time, then the decision system must decide what to produce instead. This decision system uses a sliding horizon and tries to optimize costs on any given horizon. A model and some approaches for its resolution are proposed. The optimisation criterion is the average production cost (component inventory cost and backlogging cost).
\end{abstract}

\section{Keywords}

Assembly manufacturing systems, random supply, inventory control, medium term planning, linear programming, simulation

\section{INTRODUCTION}

We consider multi-product assembly systems, which use external ordered components to assemble products. We study the medium term planning and inventory control problems when 
delivery delay of components is critical. To control such systems, the following problems arise:

- How to maintain constant levels of component inventories on the average in order to limit the consequences of random delivery delay deviations? A trade-off must be made between component inventory cost and product backlogging cost (product inventories are useless with unlimited capacity hypothesis since components not used at previous periods are used only just in time when the corresponding product is required).

- In case of insufficient component inventory level, how to manage conflicts between the products requiring simultaneously the same set of components ? The corresponding criterion value depends strongly on the maximum deviations of both component inventory cost values and backlogging cost values.

In the literature, reported works essentially deal with supplier selection strategies (Cohen and Lee, 1989; Hendrik and Rush, 1988; Trevelen and Schweikhar, 1988). In (Gurnani et al., 1990) the authors address the supplier diversification when the quantity delivered is uncertain. Ramasesh et al. (1991) have considered the randomness of the lead time but only in the case of one product and with uniform or exponential laws.

We suppose that we know the distribution laws for component delivery delays, as well as the inventory cost for each component and the backlogging cost for each product. We search a solution approach considering only steady-states for this system. So the cost is minimized only in average. If the reader is interested to know how to stabilize a transient system into a given steady-state, some approaches can be found in (Chu et al., 1994). We do not consider this problem in this paper and assume that it is possible to reach the optimal steady-state, which can be obtained by solving the model proposed.

The problem is formulated in section 2 . Then, the global solution approach is presented in section 3. Finally, some numerical examples are given in section 4 .

\section{PROBLEM FORMULATION}

In the following, we consider a steady-state of the system on an infinite horizon. Time axis is divided into a number of equal time intervals called periods. Notations used are:

- N the matrix which describes the product structure, where $N_{z}^{j}$ is the number of components $j$ required to assemble one unit of product $z, 1 \leq z \leq n, 1 \leq j \leq d$.

- $y$ the vector $\left(y_{1}, y_{2}, \ldots, y_{n}\right)$, where $y_{z}$ is the number of products $z$ to be manufactured at the end of each period.

- $r(i)$ the vector $\left(r_{1}(i), r_{2}(i), \ldots, r_{n}(i)\right)$, where $r_{z}(i)$ is the number of backloggings of product $z$ at the beginning of period $i$.

- $s(i)$ the vector $\left(s_{1}(i), s_{2}(i), \ldots, s_{d}(i)\right)$, where $s_{j}(i)$ is the inventory level of component $j$ at the beginning of period $i$.

- $\alpha$ the vector $\left(\alpha_{1}, \alpha_{2}, \ldots, \alpha_{d}\right)$, where $\alpha_{j}$ is the period inventory cost of component $j$.

- $\beta$ the vector $\left(\beta_{1}, \beta_{2}, \ldots, \beta_{n}\right)$, where $\beta_{z}$ is the period backlogging cost of product $z$.

- $\mathbf{x}(i)$ the vector $\left(x_{1}(i), x_{2}(i), \ldots, x_{n}(i)\right)$, where $x_{z}(i)$ is the number of products $z$ which are assembled during the period $i$.

- $\mathrm{q}$ the vector $\left(\boldsymbol{q}_{1}, \boldsymbol{q}_{2}, \ldots, \boldsymbol{q}_{d}\right)$, where $\boldsymbol{q}_{j}$ is the number of components $j$ ordered at the beginning of each period.

- $\mathrm{f}(\mathrm{t})$ the vector $\left(f_{1}(t), f_{2}(t), \ldots, f_{d}(t)\right)$, where $f_{j}(t)$ is the distribution law for delivery delay of component $j$. 
Let $C R_{l}(h)$ and $C R_{2}(h)$ be respectively the total inventory cost and the total backlogging cost from period $O$ to period $h-1$ included.

$$
\begin{aligned}
& C R_{I}(h)=\sum_{k=0}^{h-1} \sum_{j=1}^{d} \alpha_{j}\left(s_{j}(k)-\sum_{z=1}^{n}\left(x_{z}(k) N_{z}^{j}\right)\right) \\
& C R_{2}(h)=\sum_{k=0}^{h-1} \sum_{z=1}^{n} \beta_{z}\left(y_{z}+r_{z}(k)-x_{z}(k)\right), \\
& s_{j}(k)=s_{j}(k-l)-\sum_{z=1}^{n} x_{z}(k-1) N_{z}^{j}+\sum_{u=0}^{k-1} q_{j} d_{u, k}^{j}, \\
& d_{u, k}^{j}= \begin{cases}1 & \text { if a delivery of type } j \text { ordered at period } u \text { occurs at the end of period } k-1, \\
0 & \text { otherwise, }\end{cases} \\
& q_{j}=\sum_{z=1}^{n} N_{z}^{j} y_{z} .
\end{aligned}
$$

The goal is to minimize, $C R=\lim _{h->\infty}\left\{\frac{1}{h}\left[C R_{I}(h)+C R_{2}(h)\right]\right\}$.

\section{SOLUTION APPROACH}

\subsection{Planning model with sliding horizon}

Working on an infinite horizon is not easy, thus we decide to optimize decisions on a sliding horizon of $h$ periods. When taking $h$ equal to $l$ we obtain a simplified model called "the basic model". The following example illustrates the fact that using only the basic model does not give an optimal approach. This is due to the fact that solving optimally and successively conflicts between products for each period may be worse than solving globally conflicts between products for a series of periods even if some data are not known in advance but only forecast.

Example, $\mathbf{N}=\left[\begin{array}{ll}0 & 1 \\ 1 & 1\end{array}\right], \mathbf{y}=(2,2), \mathbf{s}(\mathbf{1})=(3,4), \quad \alpha=(10,20), \quad \beta=(30,1000), \quad \mathbf{f}_{\mathbf{j}}(\mathbf{t})=(0.5,0.5)$, $\forall \mathbf{j}=1,2$, where 0.5 is the probability of regular component delivery. We consider two periods.

Basic model: $\mathbf{x}(\mathbf{1})=(2,2) \Rightarrow C=10+0=10$.

1.1 With probability $0.5, \quad s(2)=(1,0), x(2)=(0,0) \Rightarrow C=10+2060=2070$.

1.2 With probability $0.5, \quad \mathrm{~s}(2)=(3,4), \mathbf{x}(2)=(2,2) \Rightarrow C=10+0=10$.

The average total cost $\mathrm{E}[\mathrm{C} / \mathrm{h}]=(10+2080 \times 0.5) / 2=525$.

Optimal solution: $\mathbf{x}(\mathbf{1})=(1,2)=>C=30+30=60$.

2.1 With probability $0.5, \quad \mathrm{~s}(2)=(1,1), \mathbf{x}(2)=(\mathbf{0}, 1)=>C=0+1060=1060$.

2.2 With probability $0.5, \quad s(2)=(3,5), \mathbf{x}(2)=(2,2) \Rightarrow C=30+0=30$.

The average total cost $E[C / h]=(60+1090 \times 0.5) / 2=\mathbf{3 0 2 . 5}$. 
Due to the reasons explained earlier, we decide to work with a sliding horizon which is modelled by the following model (7)-(10). The periods of a given horizon are indexed by $t_{0} t_{t}$, ..., $t_{h-1}$. For the first sliding horizon, $t_{0}$ is equal to 0 and increases by 1 at each iteration of the general solving method. For any $i=0,1,2, \ldots$, when we have to decide the number of products to be assembled, we solve this model by using integer linear programming (exact or approximation) methods with $t_{0}=i$ and we only use the solutions $\mathbf{x}\left(\mathbf{t}_{0}\right)$ associated with current period $i$.

Note that the criterion (7) is not the cost sum but a simplified form obtained by removing a constant part and by transforming a minimization into a maximization.

$$
\begin{aligned}
& \sum_{k=0}^{h-1} \sum_{z=1}^{n}(h-k) x_{z}\left(t_{k}\right)\left(\beta_{z}+\sum_{j=1}^{d} N_{z}^{j} \alpha_{j}\right) \rightarrow \max , \\
& \sum_{k=0}^{v} \sum_{z=1}^{n} \boldsymbol{x}_{z}\left(t_{k}\right) N_{z}^{j} \leq s_{j}\left(t_{0}\right)+L_{j}^{*}\left[t_{0}, t_{v}\right], \quad \forall v=0,1,2, \ldots, h-1, \\
& \sum_{k=0}^{v} \boldsymbol{x}_{z}\left(t_{k}\right) \leq(v+1) y_{z}+r_{z}\left(t_{0}\right), \quad \forall z=1,2, \ldots, n, \quad \forall v=0,1,2, \ldots, h-1, \\
& \boldsymbol{x}_{z}\left(t_{k}\right) \geq 0, \quad \forall z=1,2, \ldots, n, \quad \forall k=0,1,2, \ldots, h-1,
\end{aligned}
$$

where $L_{j}^{*}\left[t_{0}, t_{v}\right]$ is a forecast value of the components delivered within the interval $\left[t_{O}, t_{v}\right]$, $L_{j}^{*}\left[t_{0}, t_{0}\right]=0$.

It is possible to compute an upper bound of $h$ denoted by $h^{\prime}$ with the following equation:

$$
h^{*}=\max _{j}\left[\max _{z}\left(\beta_{z} / N_{z}^{j}\right) / \alpha_{j}\right], \quad \forall z=1,2, \ldots, n, \quad \forall j=1,2, \ldots, d, \quad N_{z}^{j} \neq 0 .
$$

The best experimental value of $h$ obtained by simulation (section 4) is strictly less than $h^{\prime}$ and varies with different other factors such as delivery components rate, forecasting methods, etc.

\subsection{Inventory level determination}

When some components are missing, the model (7)-(10) allows to optimally assign existing components to a limited part of the required products. Although we do not consider the transient phase, it provides us with average component inventory levels. We search for the average component inventory levels which minimize the total cost by simulation.

To obtain a good average inventory level $\omega_{j}^{*}$ for any component $j$ in the steady-state, we build an iterative algorithm. At the beginning of step $k$, the component inventory levels are given by the vector $\omega^{k}=\left(\omega_{1}^{k}, \omega_{2}^{k}, \ldots, \omega_{d}^{k}\right)$. We assume that for a small variation $\varepsilon_{j}^{k}$ of one of the component levels $\omega_{j}^{k}$, the corresponding cost variation is linear. The coefficient of the cost variation obtained with $\varepsilon_{j}^{k}=I$ is given after one simulation of $M$ periods by the equation:

$$
\gamma_{j}^{k}=1 / M\left(\sum_{i=0}^{M-1}\left(\alpha_{j} \varsigma_{j}^{i}\right)-\sum_{i=0}^{M-1} \sum_{z=1}^{n}\left(\beta_{\mathrm{z}} \psi_{z, j}^{i} / N_{z}^{j}\right)\right), \forall \mathrm{k},
$$

where $\zeta_{j}=l$ if there remains at least one component $j$ in the inventory at the end of period $i$ of the simulation and $O$ otherwise, $\psi_{z j}^{i}=1$ if at least one product $z$ is not assembled during 
period $i$ of the simulation by lack of component $j$ and 0 otherwise.

For all $j, \omega_{j}^{0}$ is obtained by uniform random generation between a lower and an upper bound (minimum and maximum delivery delay for component $\times q_{j}$ ).

At step $k$, we first simulate and compute the values $\gamma_{j}^{k}\left(\omega^{k-1}\right)$. Then we search a good value for $\varepsilon_{j}^{k}$ by an algorithm (similar to dichotomy) using the results of the above simulation and we set $\omega_{j}^{k}=\omega_{j}^{k-1}+\varepsilon_{j}^{k}\left(\varepsilon_{j}^{k}=0\right.$ when $\left.\gamma_{j}^{k}=0\right)$. The iterative algorithm stops when for all $j, \varepsilon_{j}^{k}=0$.

\subsection{Forecasting methods}

To compute forecast component future delivery $L_{j}^{*}\left[t_{0} t_{k}\right]$ for the model (7)-(10), we experiment the following methods.

Average inventory forecasting $\left(\operatorname{method} M_{1}\right)$

$$
L_{j}^{*}\left[t_{i}, t_{k}\right]=\sum_{m=i+1}^{k} \sum_{v \in \theta_{j}\left(t_{i}\right)} q_{j} \operatorname{Prob}\left\{d e l_{j}\left(t_{v} \mid t_{i}\right)=t_{m}-t_{v}\right\}
$$

where $\theta_{j}\left(t_{i}\right)$ is the set of indices of periods before $t_{i}$ where orders of components $j$ have been done, $d e l_{j}\left(t_{v} \mid t_{i}\right)$ is the conditional delivery delay of components ordered at the beginning of period $t_{v}$, knowing that this components are not yet delivered at the beginning of period $t_{i}$.

Random sample forecasting (method $M_{2}$ )

$$
\begin{aligned}
& L_{j}^{*}\left[t_{i}, t_{k}\right]=\sum_{m=i+1}^{k} \sum_{\mathrm{v} \in \theta_{\mathrm{j}}\left(t_{i}\right)} q_{j} \delta_{j}\left(t_{m} \mid t_{v}, t_{i}\right), \\
& \delta_{j}\left(t_{m} \mid t_{v}, t_{i}\right)= \begin{cases}1 & \text { if } d e l_{j}^{*}\left(t_{v} \mid t_{i}\right)=t_{m}-t_{v}, \\
0 & \text { otherwise, }\end{cases}
\end{aligned}
$$

$\operatorname{del}_{j}^{*}\left(t_{v} \mid t_{i}\right)$ is the value obtained randomly by using the distribution function laws of delivery delay of component $j$.

\subsection{Simulation model}

In order to adjust the parameters of the proposed model and control the assembly system, we designed a simulation program running under UNIX on Sun workstations. To date, the program allows to treat examples with 100 products, 300 components and 100 forecasting periods. This simulation uses the integer linear programming part of OSL software. One step of the simulation algorithm works as follows:

1. Simulate the arrival of the components.

2. Compute the conditional distribution laws for the orders whose deliveries are not arrived at the current period.

3. Compute the forecasts for future deliveries.

4. Calculate the optimum values of production using OSL.

5. Compute the new inventory levels of components.

6. Calculate the production cost. 
To obtain an ideal reference value for the average period cost, we made a series of experiments where the simulated delivery delay are taken equal to the randomly generated forecast delays. This forecasting method is called "100\% forecast" and denoted by $M_{0}$.

The following algorithm explains how to work with our model:

1. Search the best inventory level $\omega_{j}$, for all $j$.

2. Compute $h^{\prime}$ using equation (11).

3. For $h=h_{1}, h_{2}, \ldots\left(h_{l}=h^{\prime}, h_{i}>h_{i+1}, h_{i} \geq 1\right)$, use simulation with method $M_{0}$ and $s_{j}\left(t_{0}\right)=\omega_{j}$ for all $j$.

4. Keep the period length $h$ which gives the minimal total cost.

5. If $h=1$, go to 8 .

6. Apply the forecasting methods $M_{l}$ and $M_{2}$ with $h$ and keep the best one $M_{i}$ whose cost is $C\left(h, \mathbf{s}, M_{i^{*}}\right)$.

7. If $C\left(h, \mathrm{~s}, M_{i *}\right) \geq C(1, \mathrm{~s})$ then $h=1$.

8. Use sliding plan with a time $h$, the forecasting method $M_{i}{ }^{*}$ (if $\left.h \neq 1\right)$ and the quantity of components to be required $q_{j}$, for all $j$.

\section{NUMERICAL EXAMPLES}

To test the model efficiency, we have generated 5 examples. For each example, the values of matrix $\mathbf{N}$ were generated uniformly between $l$ and 10 . This provided us with different associations between the products and the components used. We took also different generated values for the other parameters of our model:

Backlogging costs: the same for all the products or very different.

- Distribution laws for delivery delays: uniform on a small interval or with one peek in the distribution law or with several peeks in the distribution law or with large standard deviation.

- Inventory levels of components: small or "optimal".

Tables 1 and 2 present the results computed for 800 periods of simulation with the following parameters, $\alpha=(10,100,1000), \beta=(10,10,10,10)$, for all $j, 3$ delay values and $f_{j}(t)=(0.333$, $0.333,0.334$ ) and inventory component levels equal to their lower bounds. In table 1:

- $\% 1$ is the maximal cost improvement (in percentage) that may be obtained by using $h$ periods instead of using the basic model. It is computed by using the ideal forecasting method $M_{0}$ (here we may see that it varies from $21 \%$ to $43 \%$ ).

- \%2 (resp. \%4) is the cost improvement obtained by using $h$ periods and the forecasting method $M_{1}$ (resp. $M_{2}$ ) instead of using the basic model (these percentages vary from $3 \%$ to $17 \%$ for $M_{2}$ and from $0,3 \%$ to $3 \%$ for $M_{1}$ which is apparently worse).

- \%3 (resp. \%5) is equal to \%2 (resp. \%4) divided by \%1 and multiplied by 100 . It represents the "true" performances obtained by method $M_{1}$ or $M_{2}$ which may be used to control on line the assembly system. Notice in table 1 that the performances vary considerably with the examples. 
Table 1 Cost comparison between forecasting methods

\begin{tabular}{|c|c|c|c|c|c|c|c|c|c|}
\hline & \multirow{2}{*}{$\begin{array}{l}\text { Basic } \\
\text { model }\end{array}$} & \multicolumn{2}{|c|}{$\begin{array}{l}100 \% \\
\text { forecast }\left(M_{0}\right)\end{array}$} & \multicolumn{3}{|c|}{$\begin{array}{l}\text { Average inventory } \\
\text { forecasting }\left(M_{1}\right)\end{array}$} & \multicolumn{3}{|c|}{$\begin{array}{l}\text { Random sample } \\
\text { forecasting }\left(M_{2}\right)\end{array}$} \\
\hline & & $C R$ & $\% 1$ & $C R$ & $\% 2$ & $\% 3$ & $C R$ & $\% 4$ & $\% 5$ \\
\hline example 1 & 8874 & 6173 & 30.4 & 8588 & 3.2 & 10.6 & 8589 & 3.2 & 10.6 \\
\hline example 2 & 7198 & 4091 & 43.2 & 7017 & 2.5 & 5.9 & 5966 & 17.1 & 39.7 \\
\hline example 3 & 7815 & 5762 & 26.3 & 7792 & 0.3 & 1.1 & 7336 & 6.1 & 23.3 \\
\hline example 4 & 10009 & 7925 & 20.8 & 9794 & 2.2 & 10.3 & 9718 & 2.9 & 14.0 \\
\hline example 5 & 7408 & 4743 & 36.0 & 7354 & 0.7 & 2.0 & 6883 & 7.1 & 19.7 \\
\hline
\end{tabular}

Table 2 illustrates the search and interest of inventory level optimization. These results were obtained with $h=1$ to limit the computation time and to obtain only one cost for each set of inventory values (if optimal inventory levels are computed with $M_{1}$ or $M_{2}$ and $h>1$, the results are quite similar to the ones obtained with $h=I$ ).

Table 2 Cost obtained with optimization of component inventory levels

\begin{tabular}{|c|c|c|c|c|c|c|}
\hline & \multicolumn{4}{|l|}{ Results of optimization } & \multicolumn{2}{|l|}{ Basic model } \\
\hline & $\begin{array}{l}\text { "Optimal" } \\
\text { inventory levels }\end{array}$ & $C R$ & $N I$ & $\% 6$ & $\begin{array}{l}\text { Lower bounds of } \\
\text { inventory levels }\end{array}$ & $C R$ \\
\hline example 1 & $360,404,180,250$ & 4193 & 264 & 52.8 & $160,160,80,120$ & 8874 \\
\hline example 2 & $190,100,310,228$ & 2761 & 75 & 61.6 & $80,50,150,100$ & 7198 \\
\hline example 3 & $280,407,230,230$ & 4028 & 143 & 48.5 & $130,170,100,110$ & 7815 \\
\hline example 4 & $442,150,356,342$ & 6288 & 59 & 37.2 & $220,150,180,160$ & 10009 \\
\hline example 5 & $261,269,360,258$ & 3660 & 126 & 50.6 & $110,110,140,110$ & 7408 \\
\hline
\end{tabular}

Where $N I$ is the number of iterations and $\% 6$ shows the percentage of gain obtained by using "optimal inventories" (from 37\% to 61\%). The "optimal" inventories are nearly twice bigger than the lower bound inventories.

\section{CONCLUSION}

A model has been proposed for the control of some multi-product assembly systems where component delivery delays are only known by distribution laws, but some other hypotheses are rather restrictive (constant product requirement and unlimited assembly capacity). A resolution approach is given which uses linear programming and simulation to improve some parameter choices. Experimentation shows the validing both of the model and the solving approach.

Introducing non constant product requirements in the model is quite obvious, for example with the hypothesis of cyclic requirements when working on an infinite horizon or non cyclic requirements on a finite horizon. In this case, a procedure must be added in the solving approach to compute at each period the component order quantities.

Introducing limited assembly capacity in the model and in the solving approach is quite obvious if anticipated production is forbidden which increases backlogging cost in average. 
Further research work must be developed to introduce anticipated production and corresponding product inventory costs in the model and in the solution method.

\section{REFERENCES}

Chu, C., Proth, J.M. and Xie, X. (1992) Supply management in assembly systems: the basic problem. Naval Research Logistic, 39, 265-83.

Chu, C., Proth, J.M., Wardi, Y. and Xie X. (1994) Supply management in assembly systems: the Case of Random Lead Times, in Proc. 11th International Conference on Analysis and Optimisation of Systems, Sophia-Antipolis, June 15th, 1994, Springer-Verlag.

Cohen, M. and Lee H. (1989) Resource development analysis of global manufacturing and distribution network. Journal of Manufacturing and Operations Management, 2.2, 81-104.

Gurnani, H.B., Akella, R. and Lehoczky J. (1990) Supply management in assembly systems General yield model, Working Paper, Graduate School of Industrial Administration, Carnegie Mellon University.

Hendrick, T.E. and Ruch, W.A. (1988) Determining performance appraisal criteria for the buyer. Journal of Purchasing and Materials Management, 24.2, 18-26.

Ramasesh, R.V., Ord, J.K., Hayya, J.C. and Pan, A. (1991) Sole versus dual sourcing i stochastic lead-time (s,Q) inventory models. Management Science, 37.4, 428-443.

Treleven, M. and Schweikhar, S.B. (1988) A risk/benefit analysis of sourcing strategies: single vs. multiple sourcing. Journal of Operations Management, 7.4, 93-114. 